\title{
Informational Psychology: A New Domain of Psychological Study
}

\author{
Guo WANG, Bi-bo XU and Zong-rong LI \\ Psychology Department, School of Education, Hubei University, China \\ *Corresponding author, zrli@hubu.edu.cn
}

Keywords: Theoretical informatics, Informational psychology, Hypothesis of "Informational Man", Principle of psychological regulation, Intelligence emergentism.

\begin{abstract}
A new discipline called "Informational Psychology" have gradually appeared in applying theoretical informatics to the study of psychology since June, 2012. It opened up a new field of psychological research, like "a new domain" of psychology to be explored. The article explains the fundamental hypotheses of informational psychology, namely informationalism, the duality of materialinformation, and material man and informational man; it illustrates the basic concepts of informational psychology, such as physical, biological, and informational psyche, living material and living information, signal thinking and symbolic thinking, etc.; it discusses the fundamental principles of informational psychology, -- that is to say the principles of structure, function, and regulation; finally it introduces the basic research methodology of informational psychology: the approach of systems science, the technique of intelligence emergentism, etc.
\end{abstract}

\section{Introduction}

With the construction, development and maturity of the knowledge system about information science, the concept of "science" has been changed from the material science (natural science) to a general term of science (i.e., the combination of material science and information science). Like "material", "information" is the fundamental scientific concept; it is the immaterial being. The results from the theoretical informatics research revealed that we live in a material and informational world; the way we think should alter from the traditional and material approach into the worldview and methodology of "the duality of material-information". It is not suitable to divide psychology into scientific and humanistic psychology any longer, because psychological phenomena contain both the material and informational feature, it is reasonable to regard those marginalized psychological subjects that focus on the informational feature of psychological phenomena as "scientific psychology". As to the traditional psychological study, including biopsychology, cognitive psychology, etc., their research objects all belong to the material feature of psychological phenomena, so we can call it "Material Psychology"; the informational feature, especially the symbolic feature of mental phenomena, requires to be interpreted by a new psychological theory, therefore, "Informational Psychology" was established; it changed the division of psychology into a new division of Material Psychology and Informational Psychology. In the "Preface" of Informational psychology: its background, essence, and application written by Zhang-ran ZHANG, professor of the philosophical school of Wuhan University, he said that informational psychology opened up a new field of psychological research, like "a new domain" of psychology to be explored [1]. This article aims to briefly introduce this fresh theoretical system of psychology. 


\section{The Fundamental Hypotheses of Informational Psychology}

\section{In a Philosophical Perspective: Informationalism}

The physicalists (or materialists) hold that the research objects of physics can be everything in the world, and everything can be completely explained by physical terms. Based on physics, materialists' psychology uses behaviorists' psychological method, employ the language of physical events, restores the psychological phenomena into physical phenomena, defines the psychological proposition as physical proposition, and then constructs the science that concerns with psychological phenomena as a branch of physical science.

Informationalism is the doctrine that is based on the information science, which insists that besides material being and its movement, immaterial being (information) and the its evolution also exist, and they cannot be explained completely by means of physics, as there are no conservation of information and informational energy; everything in the universe must be completely interpreted from the combination of material science and information science [2]. In the perspective of informationalism, the basic idea is the worldview of "the duality of material-Information", the core is the "relational model" of the connection and interaction between material and information, and the main method is information emergentism. Informationalism is the spirit of the times in the post-industrial society (Informational Society). In the history of human thought, informationalism is the fourth most profound and far-reaching thought after idealism, spiritualism, and physicalism [3].

After physicalism was widely accepted and idealism was totally denied, with the establishment and development of information science, and the popularity of computer's hardware-software thinking way, people began to agree with the "the duality of material-information" without questioning the absolutely correctness of materialism. When the study of the fundamental theory of information science made some significant achievements, informational philosophers began to claim the status and right of the informational ontology, took it as the "dualism of material-information", and identified that information is as important as material, sometimes even more important. On the basis of the "relational model of material-information " in theoretical informatics, information philosopher in the perspective of informationalism advocates that life and artificial system should be explained, designed, and tested by means of both material science and information science in order to promote the unification of sciences.

\section{Theoretical Informatics: the Duality of Material-Information}

Like "material", "information" is a fundamental scientific concept; it is the immaterial being [4]. The theoretical informatics claims that the whole universe shares "the duality of material-information"; it defines information as "the meaning of signal and symbol". Every subject in information science studies some kind of carriers of signal and/or symbol, paying attention to the "meaning" of these carriers. The subjects which study communication, computer, control, robots, etc. have the suitable definitions of information; natural science, the Humanities, and social sciences discuss the informational objects which they concern about.

The four elements that construct the whole universe can be divided into two groups: material and energy, information and intelligence. The two elements in one group are existent with each other and able to interconvert. Thus we can describe these four elements in simpler way and hold that everything in the universe shares the feature of "the duality of material-information". Material science such as physics, chemistry, and 
biology study the feature of material and energy of the objects, while information science such as cyber science, science of robots mainly focuses on information and intelligence. "Information" is "immaterial being", thus the division of material-information follows the way of "A - non A". It is a strictly scientific classification without any omission and repetition. Accordingly, we can divided science into the material science and information science.

The "duality of material-information" in mental phenomena is mainly reflected by the biological and symbolic feature of human mind. In essence, mental process is equal to informational process, especially symbolic process. Biology, neuroscience, and brain science mainly focus on the physical feature of mental phenomena, while psychology aims at the informational feature of psychological phenomena, i.e., the signal and symbolic feature of individual mentality, and the law of problem solving and program designing.

\section{Theoretical Informational Psychology: Material Man and Informational Man}

In the history of psychology, "material orientation" was dominant in the mainstream, its research object was the material carrier of mental phenomena, and it took man as the material man. According to the developmental history of psychology, it's easy to find out that, at the beginning, it was Wundt who added material into the study of psychology; then Watson from the school of behavioral psychology had extremely developed the material orientation, he abandoned "consciousness" and studied mental phenomena through observable and measurable behaviors which are caused by environmental stimuli. According to Watson's viewpoint, behavior was a kind of observable organism's reaction and was the activities of organism to deal with environment. Neo-behaviorist Skinner used animal's behavior as the research object instead of man's, proposed the theory of operant conditioning, studied the relationship between stimuli and responses by different experimental designing, and spread the research result to the advanced organism or human without verification [5]. Even though cognitive psychology which is dominant nowadays still follows this traditional path and it regards human brain as computer and considers people's cognitive activities as the operational process of computer. When the brain analyses the stimuli and the changes of the electric potential, electric wave, and magnetic field in the relative encephalic regions, the researchers stayed within the paradigm in neuroscience still pay attention to the material carrier of mental phenomena in nature.

If man is defined as material man (physical and biological man) in the viewpoint of material psychology, then the man must be informational man under the framework of informational psychology. Informational psychology prefers the "informational orientation" in psychological research. The essence of human's psychological phenomena is information while the biological materials only act as information's carriers. This is the premise and basis of all concepts and theories of informational psychology. The hypothesis of "Informational man" includes that man is both the system of material consumption and energy conversion, and the system of information processing and intelligence producing. The evolution of material life of mankind is recorded in biological gene DNA, and that of information life was kept in cultural gene MEME; Human's biology studies living material (i.e. the material life under the control of DNA), and human's psychology researches living information (i.e. the informational life under the control of MEME). Human is essentially a replication and generation system of genetic and cultural information. The reason why the people can be called as human beings is that people not only possess reason to produce and use tools, but also create and employ symbols to develop cultural and science system. Human activity 
requires not only the support of physical force and the provision of genetic program, but also the provision of social cultural program, such as the private schedule or state plans.

\section{The Basic Concepts of Informational Psychology}

\section{Physical, Biological and Informational Psyche}

In each period of science history, a kind of science which was always in a dominant position impacts and constraints the development of other disciplines. Dating back to the development of science, we can find that there are three typical leading sciences: physics, biology, and informatics. Separating the form of faith and speculation, psychology was in turn under the control of the worldview and methodology of these three leading sciences respectively at the stage of empirical research.

When physical methods were popular, Wundt and other psychologists abandoned the study of speculative psychology and began to use the worldview and methodology of physics to research scientific psychology, then psychology had became a branch of physics and psychophysics was created. In 1860s, Fechner published the famous book Elemente der Psychophysik (Element of Psychophysics) defining psychophysics in the form of subtitle as "a function of psychosomatic discussion or exact sciences of mutual relations", which was the symbol of the establishment of the classic psychophysics.

The traditional biology is a category of natural science that focuses on the structure, function, occurrence, and development of biology and the relationship between biology and environment. In view of the uniqueness of people's psychological phenomena and its dependence on biology, it was inevitable to study the biological basis of people's psychological phenomena in the development of psychological disciplines, so that psychology naturally became a branch of biology. Psychobiology is a scientific area which contains the study of biological basis for the behavior and mental activity. It attempts to explain the psychological phenomena in accordance with the biological basis. Among them, the biological basis of psychological phenomena is a recurring theme in psychological history.

With the development of the age and the impact of informational revolution, "information" has become an indispensable and important theme in our life; at the same time, with the popularity of the concept of the duality of material-information, psychology as a branch of Informatics has become the inevitable trend of scientific development. Strictly, psychological informatics (i.e. informational psychology, their semantics are the same, but their pragmatics are different very little) is the result of the popularization and application of theoretical informatics in theoretical psychology and fundamental psychology, and can be considered as the test and verification of theoretical informatics. Theoretical informatics considers that each object in psychological phenomena has both material feature and informational feature by applying the information emergentism and taking the psychological information as the fundamental research content, which easily overcomes the contradictions and defects in the traditional psychology, and provides a new direction and viewpoint for further research and development of psychology. Informational psychology acknowledges the validity and necessity of "classic" psychology, absorbs the viewpoint and method of traditional psychology, develops itself, and gradually forms its own theoretical basis and knowledge system; it can be said that informational psychology impacts the spirit of the post-industrial times, and keeps pace with the age of information. 


\section{The Living Material and Living Information}

Information can be divided into living information and non-living information, living information can be split into human and non-human information, and the human information can be separated into physical and psychological information. Due to the physical information from DNA, human becomes a living physical system; and because of cultural gene (MEME), human becomes a living information system [6]. Human possesses the metabolic functions of material and energy, but we cannot produce them, however, human can directly produce knowledge and intelligence. That's the meaning and value to be alive.

Theoretical informatics supports the hypothesis of informational man, regards "man" as a "living" informational system which has multi-layer network structure (including physiological and psychological information systems), and is good at copying and creating something; human's hardware, which is the "living" material physiological system, plays the supporting role in the survival and development of informational system. The nature of human being is not like a wine skin and rice bag, but a living and creative system of signal and symbol. When one man's life is going to the end, his body may return to the nature, but his spiritual production will remain forever. The research result of theoretical information scientists may lead to some kind of particularity of their worldview, philosophy, and value.

\section{The Informational Thinking and Symbolic Thinking}

Signal and symbol are the two carriers of human's perception, imagination, learning, memory, and thinking. Before the invention of symbol, our ancestors didn't possess symbolic awareness and thought, they only had signal awareness and thought like existent animals. Today human with culture and civilization has animal's signal awareness and his own symbolic awareness. Symbol is the signal of signal, or the second signal in another word; it is a kind of signal, namely the signal that has been symbolized.

In brain science, paleocortex is called as "reptile's brain", which controls animal's muscle, balance, and auto functions like breath and heartbeat. This part of animal's brain keeps activating, even in deep sleep status. Neocortex of human brain, namely advanced brain or rational brain, gives pallium the function of cognition, making human stick out from animals. Hence, human possesses not only the first signal system like other animals which can reply for objective things, but also the speech center (including listening, speaking, writing, and reading) as the nerve of action of the second signal system, using the second signal system--words (including language and thinking with words) to summarize and to carry out an abstract activity.

Information scientists believe that we can logically divided human brain into signal brain and symbolic brain; the former has created symbol, and finally the application and accumulation of symbol have shaped the latter. It can be said that human's signal awareness creates symbolic awareness and signal thought is the father of symbolic thought.

\section{The Fundamental Principles of Informational Psychology}

\section{The Structural Principle}

The structural principle of informational psychology mainly studies the composition and structure of human psychic system. Psychic system mainly includes the following three aspects: motivation, knowledge and wisdom. Among them, the motivation is the engine to inspire, guide, and support the activities of individual; each activity of 
individual will have one motivation and different activities have different motivations, which give us different directions. Knowledge is our accumulated wealth during the process of understanding the world and can be a reference to new problem and situation, but as time goes by, knowledge can be changed both in quality and quantity. The reason why wisdom is uniquely linked to human is because what you need is wisdom if you really want to do one thing, although motivation can excite individual actions and knowledge can provide information of how things can be done. But One of the most important aspects attaining wisdoms is to find the law of everything, the other is to find the method of doing things. The combined action of motivation, knowledge and wisdom decides the direction and quality of people's action. These three elements can be considered as a triangle, in which motivation, knowledge, and wisdom are the vertices, among which motivation is at the top and knowledge and wisdom are at the bottom. The triangle may be divided into two parts, as shown in figure 1 below.

Motivation

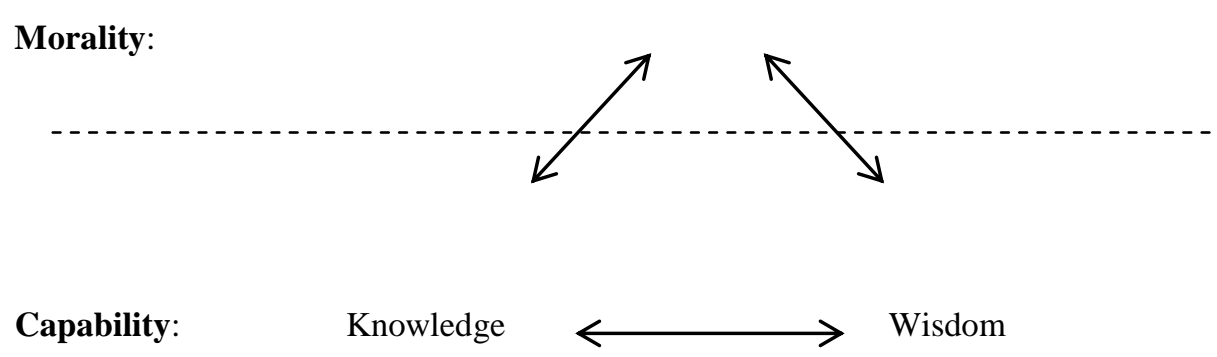

Figure 1. The structure of informational psyche

\section{The Functional Principle}

Informational psychologists point out that human psychological system has nine fundamental functions, namely sensation, perception, attention, imagination, language, learning, memory, introspection and thinking. These functions play their own unique and important role, and they work together to make people grow and mature constantly. Based on sensation and perception, people get a certain degree of knowledge and experience. These knowledge and experience become thinking by indirect generalization. The content of thinking should be communicated with people through language, and be processed into imagination. The knowledge and experience will be memorized through accumulation and preservation. The whole mental activity must be under the premise of attention. After a period of time, people's psyche will have a lasting changes, which is reflected in the learning process and accompanied with introspective process. Each stage is essential, and they make people a perfect and unified whole.

\section{The Regulating Principle}

Informational psychologists suggested that the regulating principles of human mind system include the passive adjustment mechanism of animal's "environmental stimuli and subject's reaction", the initiative control mechanisms of the public "problem solving and program designing", and the self-development mechanism of elites' self-perfection and self-actualization. These regulating principles are developed from lower level to higher one: the first is that both animal and human have the first regulating mechanism to maintain the fundamental survival needs; the second 
mechanism aims to seek solutions to current problems which is unique to human, and the third one is only popular among a few of the "elite". These elites regard self-realization as the highest goal, hope to achieve their full potential, and obtain the sense of satisfaction by creating their "brilliant life". In conclusion, these three mechanisms play an important role in our life; when the situation and the requirements alter, different people will have different reactions.

\section{The Fundamental Research Methodology of Informational Psychology}

\section{The Approaches of Systems Science}

In 1860, Fechner announced his research result by publishing the book Elemente der Psychophysik (Element of Psychophysics). Wundt established his psychological lab in which he shared the similar idea of Fechner's; Essentially, the idea could only focus on those "single elements" in the lower level of mental phenomena like "sensation" as being limited into Newton's "approach of scientific philosophy for single particle", so it's impossible for it to study the advanced mental process. Everything in the world can be seen as a system, thus the systems are ubiquitous. The whole structure and function of human's mental system can only be revealed by the means of system theory, especially that of informational system theory. The meaning of holism's principle of systems science is clearer than the holism in other philosophy, for which one example is the well-known view point that "the whole is greater than the sum of the parts".

The approach of system theory is the application of the principle of system theory. Systems scientists investigate the object comprehensively and accurately in the form of systematic thinking, by exploring some connection and interaction between system and element, structure and function, and system and environment, so that the problems can be solved in most suitable way. The fundamental principles of system method are the principles of integrity, relativity, dynamic state, comprehensiveness, and optimization.

The approach of information is the core and soul of system scientific methodology, as each complicated system contains a informational system which manages and regulates the material system. The functions of the informational approach are as following: (1) Revealing the informational connection among the material motion of robot, biological organism, and the society. (2) Revealing the new law of some events' motion and giving scientific explanation on those tough phenomena. (3) Supplying the thinking way to fulfill the modernization of scientific technology, production, operating management, and social management.

\section{The Techniques of Intelligence Emergentism}

When we look at psychology from the perspective of theoretical informatics, its basic method must be associated with the method of information science. The main method of theoretical informatics is information emergentism. With combining the advantages of quantitative and qualitative approaches in traditional psychology and absorbing the feature of methods of information science, the uniquely fundamental method of informational psychology is formed: the intelligence emergentism.

Emergence refers to the phenomena that an individual in the system follows simple roles, and that some new attributes or rules will appear in the system when the individual constitutes a whole by interaction of local parts [7]. Emergentism is based on the individual's law, but the law of the whole cannot be explained perfectly by the individual's law; As to this difficulty of the explanation, it was said that the whole is greater than the sum of the parts in a system. The nature of emergence could be described as following: (1) An emergence appears, a set of simple rules are needed, and the parts can interact with each other in the local environment; (2) The parts in the 
system must be nonlinearly interacted, and those systems that can be explained by reductionism are impossible to have any emergent phenomenon.

About the universal components, the history of human thought has experienced a development process from monism to binary, ternary, and quaternary theory. Early monists believed that the world was made up by material phenomena, and dualists further proposed the concept of energy with the development of the times, then the ternary theorists put forward the informational phenomena based on them. We believe that the energy in dualism corresponds to the material energy in essence, therefore informational energy should be added into the universal components to form a quaternary theory: matter, material energy; information, informational energy. As to this informational energy, it is just the "intelligence" we said in the system of human psychology, and the "artificial intelligence" we called in a computer and a robot.

In figure 1, the morality, namely the motivation, is man's value, which is regarded as "switch" to determine the direction of people's thought and behavior. The ability contains two parts: knowledge and wisdom. The knowledge (perceptual and conceptual) supports man's thinking as "fuel"; The wisdom acts as the engine, following the break-collect rule, forming the ability to analyze and solve problems, emerging eventually intelligence, fulfilling the function of psychological system, and guiding people's actual behavior specifically. The leading factor and the core of informational psychological research is the emergent mechanism of human intelligence.

\section{Summary}

At present, the development of modern psychology which is dominated by material psychology meets its barrier, while it is very hard to overcome the problem by the traditional means of psychology. We believe that the concept of "the duality of material-information" can be combined with the research results from different schools, that informational psychology is the key to solve the problem, and that intelligence emergentism can run through the entire procedure and different aspects of man's mental process. Like the relationship of isolation and complement between hardware and software of a computer and between physiological subsystem and psychological subsystem of human brain, the relation between material psychology and informational psychology includes isolation and complement. If we accept informational psychology as a new domain of psychology and like the "informational orientation" being claimed by informational psychology, we can step on this "new domain" of psychology to move forward under the guidance of the spirit of the age of information.

\section{Acknowledgement}

We thank Prof. Zheng-qing DAI, Vice Director of the Institute of Developmental and Educational Psychology in Wuhan university, Dr. Ling XIAO from the Mental Health Center in Renmin Hospital of Wuhan university for their supporting and taking part in the interdisciplinary research of "Informational Psychology" dominated by Prof. Zong-rong LI. We are grateful to Prof. Guo-cai YAN from the psychology department of Shanghai Normal university, Prof. Liang-shi YAN from the psychology department of Hunan Normal University, and Prof. Zhang-ran ZHANG from the psychology department of Wuhan University for their guidance and support to the research. 


\section{References}

[1] Zong-rong Li, Ling Xiao, Informational Psychology: the Background, Essence, and Application. Wuhan University Press, Wuhan, 2015.

[2] Zong-rong Li, Theoretical Informatics: Concepts, Principles, and Methodology, doctorial dissertation. Huazhong University of Science and Technology, Wuhan, 2004.

[3] Zong-rong Li, Mario Bunge, On the World-view of Information Science. J. Medical Information. 21(2008)1223-1234.

[4] Gary E. Kessler, Voices of Wisdom----A Multicultural Philosophy Reader, ninth edi., Wadsworth Publishing House Company, California, 2015.

[5] David G. Myers, Exploring Psychology, fifth edi., Worth Publishers, New York, 2006.

[6] Zong-rong Li, An Introduction to Theoretical Informatics, China Science and Technology Press, Beijing, 2010.

[7] Mario Bunge, Emergence and Convergence----Qualitative Novelty and the Unity of Knowledge, University of Toronto Press, Toronto, 2003. 